\title{
Molecular Mechanisms of Trastuzumab Resistance in HER2 Overexpressing Breast Cancer
}

\author{
Gabriel L. Fiszman and María A. Jasnis \\ Immunobiology Department, Institute of Oncology A. H. Roffo, University of Buenos Aires, Avenida San Martín 5481, \\ CP1417 DTB Buenos Aires, Argentina \\ Correspondence should be addressed to Gabriel L. Fiszman, gfiszman@gmail.com
}

Received 30 April 2011; Accepted 1 July 2011

Academic Editor: Alejandro J. Urtreger

Copyright ( 2011 G. L. Fiszman and M. A. Jasnis. This is an open access article distributed under the Creative Commons Attribution License, which permits unrestricted use, distribution, and reproduction in any medium, provided the original work is properly cited.

The epidermal growth factor receptor 2 (HER2) is a tyrosine kinase overexpressed in nearly $20 \%$ to $25 \%$ of invasive breast cancers. Trastuzumab is a humanized monoclonal antibody that targets HER2. The majority of patients with metastatic breast cancer initially respond to trastuzumab, however, within 1 year of treatment disease progresses. Several molecular mechanisms have been described as contributing to the development of trastuzumab resistance. They could be grouped as impaired access of trastuzumab to HER2, upregulation of HER2 downstream signaling pathways, signaling of alternative pathways, and impaired immune antitumor mechanisms. However, since many of them have overlapping effects, it would be of great clinical impact to identify the principal signaling pathways involved in drug resistance. Significant efforts are being applied to find other therapeutic modalities besides trastuzumab treatment to be used alone or in combination with current modalities.

\section{Introduction}

Breast cancer is the most common malignancy worldwide and the second leading cause of cancer death in women in USA and the first one in Argentina [1]. In the past decades, the development of strategies for breast cancer treatment focused on understanding the expression, regulation, and function of critical signaling pathways involved in cancer initiation and progression and allowed the identification of breast cancer subsets with different biology [2]. One of the most important targeted therapies has been the use of the antihuman epidermal growth factor receptor 2 (HER2) for tumors overexpressing this receptor [3].

Breast cancer is a heterogeneous disease that can be classified in different subsets with distinct biology and molecular profiles [4], some of which can be associated with enhanced tumor aggressiveness and poor clinical outcome [5].

Breast tumors vary according to the expression of estrogen receptor $(\mathrm{ER})$, progesterone receptor $(\mathrm{PR})$, and amplification of HER2 which is overexpressed in approximately $20 \%$ to $25 \%$ of invasive breast cancers [6]. The resulting subgroups are important not only for clinical behaviour and prognosis, but also for predictive response to targeted therapies against these receptors and the pathways they activate.

The HER2/neu gene encodes a 185-kDA transmembrane tyrosine kinase (TK) receptor that belongs to the EGF receptor (EGFR) family which consists of EGFR/ErbB1 (HER1), ErbB2 (HER2), ErbB3 (HER3), and ErbB4 (HER4). All receptors, with the exception of HER3, contain a cytoplasmatic TK region and all, with the exception of HER2, bind specific ligands via extracellular domains. Upon ligand binding, receptors dimerize using HER2 as their preferential binding partner [6]. These receptors are expressed in a variety of tissues of epithelial, mesenchymal, and neuronal origin [7]. Activation of the HER receptors under physiological conditions is controlled by spatial and temporal expression of their ligands, members of the EGF family of growth factors. Homo- or heterodimerization of receptors after ligand binding results in the phosphorylation of residues from the intracellular domain of the receptor, resulting in the recruitment of signaling molecules from the cytoplasm and the initiation of several signaling pathways. HER2 dimerization triggers diverse cellular processes related 
to enhanced cell motility, survival, and proliferation as well as resistance to apoptosis [8]. Certain pathways are preferentially modulated by different receptors due to the ability of each receptor to bind specific effector proteins. Two of the main activated pathways are RAS/Raf/MAPK and the phosphatidylinositol 3-kinase (PI3K)-Akt pathways [9].

Overexpression of HER2 enables constitutive activation of growth factor signaling pathways, serving as oncogenic drivers in breast cancer. It is well known that cancer patients whose tumors have alterations in HER receptors tend to have a more aggressive disease associated with predictive factors of a poor clinical outcome [10]. Constitutive EGFR activation can be elicited by EGF-related growth factors produced either by the tumor cells themselves or by the surrounding stromal cells [11].

\section{HER2 as Target for Cancer Therapy}

Amplification of HER2 was originally detected in a subset of breast tumours [12] as well as in gastric [13], ovarian [14], and salivary gland cancers [15]. Concerning breast cancer treatment, HER2 has features that allow it to be considered as an ideal therapeutic target, since their levels strongly correlate with tumorigenesis, as demonstrated by gain [16] and loss of function [17], gene overexpression in metastatic breast cancers [12], and its association with poor diseasefree survival [18]. Overexpression (25-fold increase in HER2 gene copy numbers) is found in nearly $30 \%$ of breast cancer [19] either in primary tumors as well as in metastatic sites, indicating that anti-HER2 therapy would be an effective approach for all disease sites. Hence, targeting HER2 protein could reduce the pathogenicity caused by tumor cells [20].

Two important types of HER2 inhibitors are currently in clinical use: humanized antibodies directed against EGFR/ErbB2 and small-molecule tyrosine-kinase inhibitors (TKIs) that compete with ATP in the tyrosine-kinase domain of the receptor. In preclinical models, both inhibitors rapidly downregulate PI3-AKT, MAPK, SRC, and STAT signaling, consequently blocking the proliferation of tumour cells and human xenografts in nude mice $[21,22]$.

Antibody-based therapy targeting HER2 is based on the development of monoclonal antibodies (mAbs) against epitopes present in the HER2 extracellular domain. Upon binding to their cognate epitopes, these antibodies exert their antitumor effects by multiple mechanisms.

Trastuzumab (Herceptin, Genentech Inc., San Francisco Calif, USA) is a humanized recombinant $\mathrm{mAb}$ that binds to the extracellular domain of the human HER2 protein [23]. Currently, it is the only HER2-targeted therapy approved by the FDA for metastatic breast cancer treatment. It selectively exerts the antitumor effects in experimental animals and patients with HER2-amplified breast tumors but not in tumors with normal HER2 expression [24, 25]. However, the clinical efficacy of trastuzumab is limited, since a significant number of patients with HER2 overexpressing tumors will be initially or eventually resistant to trastuzumab [26]. An overall response rate (complete + partial responses) ranging from $15 \%$ to $30 \%$ has been obtained when used as single agent; however, higher response rates (50\%-80\%) have been reported when used in combination with standard chemotherapy for metastatic disease [27-29].

Understanding the mechanisms of resistance to trastuzumab is, therefore, crucial for the development of new therapeutic strategies [7].

\section{Mechanisms of Trastuzumab Action}

Although trastuzumab is currently widely used for the treatment of HER2-overexpressing breast cancer, its mechanism of action is not fully understood [30].

3.1. Effect on Cell Cycle. Trastuzumab binds to HER2 with high affinity, inducing a cytostatic effect associated with G1 arrest, reduction in cell proliferation, and apoptosis via upregulation of the cyclin-dependent kinase inhibitor p2 $7^{\mathrm{kip} 1}$ [31]. It has been demonstrated by Western blot and by immunoprecipitation that breast tumor cell lines treated in vitro with trastuzumab increased p2 $7^{\text {kip } 1}$ levels and interaction with CDK2, resulting in a decrease in CDK2 activity [32].

3.2. Effect on the PI3K Pathway. HER2 and EGFR activate signaling through PI3K/Akt pathway. When PI3K becomes activated, it generates phosphoinositides which cause AKT translocation to the plasma membrane, where it phosphorylates and becomes able to phosphorylate different targets. On the other hand, AKT can be negatively regulated by antagonizing the action of the phosphatase and tensin homologue (PTEN) on PI3K. Trastuzumab can possible reduce the signaling by this pathway, promoting arrest of cell proliferation and apoptosis [33].

3.3. Inhibition of HER2 Extracellular Domain Proteolysis. Overexpressed HER2 undergoes proteolytic cleavage, releasing the extracellular domain which generates a truncated membrane-bound fragment (p95) found in serum patients [34]. Molina et al. [35] demonstrated that trastuzumab inhibited HER2 cleavage and thus the shedding of the extracellular domain, possibly by the inhibition of metalloproteinases activity. Indeed, the authors detected a phosphorylated truncated receptor in almost half of the human breast cancer serum samples. A decrease in serum HER2 extracellular domain upon trastuzumab treatment could be considered as predictor of host response [36], since it was associated with improved progression-free survival.

3.4. Inhibition of Angiogenesis. Trastuzumab modulates the effects of different pro- and anti-angiogenic factors as well as normalization and regression of the vasculature in a xenograft model of human HER2-overexpressing breast tumor. A reduction of VEGF production only in vitro has been described, indicating that in vivo, the effect of trastuzumab is VEGF-independent [37]. A decrease in microvessel density in vivo has also been observed [38]. Expression of proangiogenic factors was reduced, while expression of antiangiogenic 
factors was increased in trastuzumab treated tumor-bearing animals versus controls.

3.5. Internalization and Degradation of HER2 Protein. Another mechanism of trastuzumab effect can be due to downregulation of HER2 through endocytosis; clinical data confirming this pathway is conflicting. Hommelgaard et al. [39] using the breast cancer cell line SKBR3 described the preferential association of HER2 with the plasma membrane protrusions, making HER2 an internalization-resistant receptor. Cuello et al. [40], using the same tumor cell line, reported that trastuzumab downregulated HER2 protein. The formation of large lattices between antibodies and antigens at cell surface, collapsing into the cytoplasm and being degraded in lysosomes has been proposed [41]. In this case, internalization and degradation would be increased by the concomitant use of antibodies targeting different epitopes on HER2, as shown by Portera et al. [42].

3.6. Immune-Mediated Response. Data from in vivo experiments and clinical trials indicate that the efficacy of trastuzumab could be partially related to its induction of an immune response. In vivo breast cancer models and clinical trials have demonstrated that trastuzumab has not only cytostatic but also cytotoxic properties, at least in part via antibody-dependent cellular cytotoxicity (ADCC). This immunological effect has been observed in vitro with a variety of breast cancer cell lines [43]. Stockmeyer et al. [44] demonstrated that apoptosis and ADCC against the breast cancer cell line SKBR3 treated with bispecific anti-HER2 $\mathrm{mAb}$ only happened when neutrophils were present.

It is known that ADCC is mainly due to natural killer cells (NK) that, when activated, express the $\mathrm{Fc} \gamma$ receptor that binds the Fc domain of the IgG1 trastuzumab. In a xenograft model, activated NK could lyse tumor cells associated with a tumour regression rate of $96 \%$. In mice lacking the Fc receptor, only $29 \%$ tumour growth inhibition was observed [44]. These results led to the conclusion that an active immune response to trastuzumab may be responsible for the antitumor activity. Gennari et al. [45] selected 11 patients with HER2-positive breast tumours receiving trastuzumab at a standard dose before breast surgery. Trastuzumab treated patients who showed complete or partial remission were found to have a higher in situ infiltration of leukocytes and a higher capability to mediate in vitro ADCC. However, they could not detect changes in total HER2 levels or downstream signalings such as alterations in cell proliferation or tumor vasculature. It is necessary to continue studying the participation of ADCC in mediating the response to trastuzumab.

3.7. Inhibition of DNA Repair. Pietras et al. [46] observed that trastuzumab partially inhibited DNA adducts repair induced by cisplatin and that it also blocked DNA synthesis after radiation in in vitro studies [47].

\section{Mechanisms of Trastuzumab Resistance}

It should be noted that most of the mechanisms of trastuzumab resistance have been identified in preclinical models and have not been completely validated in clinical samples. One important goal is to clarify which of all the mechanisms are clinically relevant. However, it is known that, as with other drugs, clinical resistance will be multifactorial. [7, 30, 48].

The most relevant mechanisms of trastuzumab resistance in breast cancer are shown in Figure 1.

\section{Impaired Trastuzumab Binding to HER2}

5.1. Truncated HER2. Different metalloproteases cleave the extracellular domain of HER2, creating a truncated receptor to which trastuzumab is unable to bind [49]. Thus, the membrane-bound portion, remains as a truncated constitutively active HER2 receptor with kinase activity but lacking the extracellular domain to bind trastuzumab [50]. However, in vitro data suggested that trastuzumab could block the cleavage of HER2 and consequently the production of constitutively active p95HER2 [35]. A retrospective clinical study revealed a strong association between the presence of p95HER2 and patients resistance to trastuzumab treatment [50]. Extracellular domain HER2 level has been evaluated as potential predictor for treatment response in metastatic breast cancer but had low predictive values for clinical benefit with trastuzumab therapy [51]. According node status and HER2 expression, Molina et al. [49] reported that the expression of p95 HER2 was more frequent in node-positive cases compared to node-negative ones.

5.2. Masking with MUC 4 and CD44/Hyaluronan Polymer Complex. Epitope masking has also been described as a mechanism of resistance to trastuzumab. Mucin 4 (MUC4) is membrane-associated glycoprotein [52], which consists of several highly glycosylated proteins that form protective barriers on epithelial cells including mammary epithelium. In this sense, MUC4 can contribute to cancer progression due to its ability to inhibit recognition of cancer cells by the immune system, promoting tumor progression, metastasis, and suppression of apoptosis [53, 54]. MUC4 may interfere with trastuzumab binding by masking HER2 receptor [55, 56]. In a preclinical study with the human HER2-positive trastuzumab-resistant JIMT-1 cell line, expression of MUC4 was associated with decreased antibody-binding capacity, being able to reverse resistance using RNA interference to knockdown MUC4 [56].

Another HER2 masking that blocks trastuzumab binding is mediated by CD44, a transmembrane receptor for hyaluronan. It has been shown that binding of polymeric hyaluronan, activates CD44-mediated signaling pathways including RAS and PI3K [57]. Binding of endogenous hyaluronan polymer to CD44 contributes to PI3K/Akt activation [58]. Blocking CD44- hyaluronan polymer binding by the use of anti-CD44 antibodies or hyaluronan oligomers suppressed the PI3K/Akt signaling pathway, inhibiting anchorage-independent tumor cell growth. 


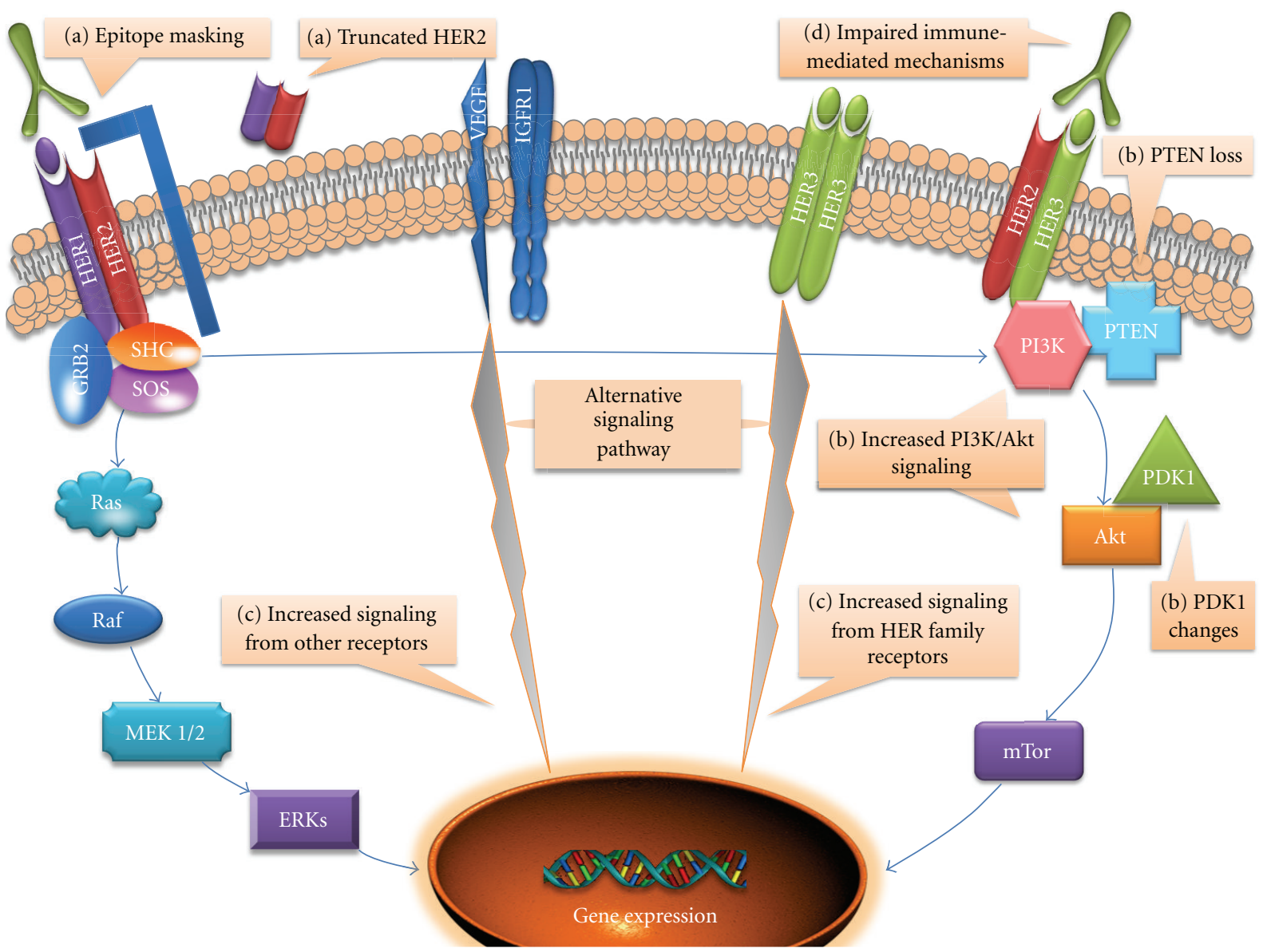

FIGURE 1: Relevant mechanisms of trastuzumab resistance in breast cancer: (a) Impaired trastuzumab binding to HER2: truncated HER2 and epitope masking. (b) Upregulation of HER2 downstream signaling pathways: PTEN loss, increased PI3K/Akt activity and PDK1 changes. (c) Alternative signaling pathways: Increased signaling from HER family and other receptors. (d) Impaired immune-mediated mechanisms.

\section{Upregulation of HER2 Downstream Signaling Pathways}

6.1. PTEN Loss. The loss of function of PTEN (phosphatase and tensin homolog) caused by mutation, or by transcriptional regulation, has been described in several tumors including nearly $50 \%$ of breast cancers [59]. PTEN normally inhibits the activation of PI3K; therefore, PTEN loss results in constitutive activation of PI3K/Akt [60]. Decreased expression or activity of PTEN blocked trastuzumab-mediated growth inhibition in HER2 overexpressing breast cancer cells [61]. Nagata et al. [33] have emphasized the important role of decreased expression of PTEN protein resulting in an increase in PI3K/Akt phosphorylation and signaling, preventing trastuzumab-mediated growth arrest of HER2-overexpressing breast cancer cells. Patients with PTEN-deficient HER2-overexpressing metastatic breast cancer had significantly worse responses to trastuzumab than those with normal PTEN tumors [33, 59]. Berns et al. [62], using a large-scale RNA interference screen, identified PTEN as the only gene whose knockdown resulted in trastuzumab resistance.
In clinical trials, Dave et al. [63] confirmed that low PTEN or activating mutation in PIK3CA conferred resistance to trastuzumab regimen, whereas low PTEN tumors were associated with a high pathologic complete response. Activation of PI3K pathway was associated with trastuzumab resistance, whereas low PTEN predicted for response to lapatinib. These observations support clinical trials with the combination of both agents.

6.2. Increased PI3K/Akt Activity. PI3K mutations are also involved in trastuzumab resistance through PI3K/Akt pathway activation. The PIK3R1 gene encodes the PI3K regulatory subunit $\mathrm{p} 85 \alpha$, affecting its function and inducing constitutive activation of the PI3K/Akt pathway $[64,65]$. In addition, PIK3CA (activator of the same pathway and frequently mutated in breast cancer) is the gene that encodes the catalytic subunit of $\mathrm{p} 100 \alpha$ of PI3K. It is frequently mutated or overexpressed in human cancer [66] and is able to confer resistance to trastuzumab in cell culture [66]. In a clinical study using 55 patients with breast cancer, Berns et al. [62] described significantly improved ability to detect 
patients with low response to trastuzumab when combining the analysis of low PTEN expression with the presence of oncogenic PIK3CA mutations. They also described an association of PIK3CA hotspot mutations and reduced time to progression after therapy.

Junttila et al. [67] showed that trastuzumab disrupts ligand-independent ErbB2/ErbB3/PI3K complexes blocking AKT signaling; when PI3K is mutated, complex disruption does not inhibit AKT, explaining why trastuzumab is ineffective in some tumors. Recent studies using HER2-amplified breast cancer cell lines showed that those cell lines with PIK3CA hotspot mutations were significantly more resistant to trastuzumab than those without mutations [68]. Thus, assessment of PI3K pathway activation might be considered a biomarker to identify patients unlikely to respond to trastuzumab-based therapy. An activating mutation of Akt1 situated in the pleckstrin homology domain (E17K) was identified in breast cancer and occurs early in the development of the disease [69]. However, this mutation has not been identified in HER2-overexpressing tumors [70].

6.3. PDK1 Changes. Overexpression of PDK1 was found in approximately $20 \%$ of breast cancers [71]. Tseng et al. [72] in a preclinical model reported that the combined use of PDK-inhibitors with trastuzumab reversed the trastuzumabresistant phenotype of SKBR3 human breast cancer cells.

6.4. Modulation of $p 27^{\text {Kip } 1}$. Another mechanism of trastuzumab resistance is dependent of the cyclin-dependent kinase inhibitor $\mathrm{p} 27^{\mathrm{kip} 1}$ [73]. Several studies have suggested that $\mathrm{p} 27^{\mathrm{kip} 1}$ is a critical element of trastuzumab response and that its downregulation can promote trastuzumab resistance [74]. The growth inhibitory properties of trastuzumab depend, in part, upon the effects on the cyclin-dependent kinase-inhibiting protein $\mathrm{p} 27^{\mathrm{kip} 1}$ [75]. Treatment with cyclin-dependent kinase 2 (CDK2) inhibitors led to a dramatic decrease in cell proliferation and enhanced apoptosis [76]. In vivo, CDK2 inhibition significantly reduced tumor growth of trastuzumab-resistant xenografts. They suggested that the use of CDK2 inhibitors in patients with breast tumors with HER2 and cyclin E coamplification/overexpression might be a valid strategy. Le et al. [77] have also suggested that any drugs or therapies that directly affects p $27^{\mathrm{kip} 1}$ or its signaling would influence the efficacy of trastuzumab. Actions on more than one target or pathways may have synergistic or additive effect against overexpressing HER tumor cells.

\section{Alternative Signaling Pathways}

Signaling through multiple alternative pathways has been linked to trastuzumab resistance in various preclinical models.

7.1. Increased Signaling from HER Family Receptors. Compensatory crosstalk occurs among receptors within the HER family [78-80]. Although trastuzumab reduces HER2mediated signaling, it might not reduce signaling from other HER receptors. HER1/HER3 heterodimers or HER1/HER1 homodimers expressing cells might initiate MAPK and PI3K signaling even in the presence of trastuzumab $[21,78]$.

HER3 is the favored receptor for dimerization with HER2 [81], and growing evidence supports HER3 as being a required partner in HER2-overexpressing breast cancer $[82,83]$. The oligomerization properties of the HER receptors confirmed that interactions between EGFR/HER2 and HER2/HER3 are detected in the presence of ligand. In addition, they found that trastuzumab is ineffective in blocking HER2/HER3 dimerization [84].

Transforming growth factor $\alpha$ (TGF $\alpha$ ) is a ligand of the HER family, and it is involved in the formation of HER2 heterodimers. In vitro, trastuzumab was less efficient in inhibiting cell growth in presence of TGF $\alpha$ [85]. In addition, increased levels of the HER family ligands such as heregulin and EGF blocked trastuzumab-mediated growth inhibition in HER2 overexpressing breast cancer cell lines [86].

\subsection{Increased Signaling from Other Receptors}

7.2.1. Insulin-Like Growth Factor-1 Receptor Overexpression. The insulin-like growth factor-1 receptor (IGF-1R) is a transmembrane TK receptor frequently expressed in human breast cancers. Its downstream effectors promote proliferation and metastatic dissemination [30]. HER-2 and the IGF-1R share common postreceptor signaling pathways. Cell proliferation is also stimulated by IGF-1R which interacts with HER2 in trastuzumab-resistant cell lines. The first research article that focused on molecular mechanisms contributing to trastuzumab resistance described IGF1R signaling as the contributing cause of resistance [87]. The authors demonstrated that trastuzumab-mediated growth inhibition was lost in breast cancer cells that overexpressed both HER2 and IGF1R. Preclinical studies have implicated IGF-1R-signaling in resistance to trastuzumab and anti-IGF$1 \mathrm{R}$ drugs restored sensitivity in resistant cells [88]. IGF$1 \mathrm{R}$-mediated resistance to trastuzumab seems to involve the PI3K/Akt pathway, leading to enhanced degradation of p27 $[48,89]$. It can be suggested that coexpressing HER2 and IGF-1R tumors are more likely to be resistant to trastuzumab therapy.

\subsubsection{Vascular Endothelial Growth Factor Overexpression.} Increase in tumor angiogenesis in breast tumors is associated with overexpression of HER2 [90]. Mice treated with trastuzumab induce normalisation and regression of the vasculature in a xenograft model of HER2-overexpressing breast tumour. Izumi et al. [37], however, observed a reduction in vascular endothelial growth factor (VEGF) production by cancer cells only in vitro, indicating that a VEGFindependent antiangiogenic mechanism might act in vivo. This mechanism appears to be due to the modulation of different regulators of the complex machinery of angiogenesis.

7.2.3. c-Met Coexpression. The c-Met receptor, another TK receptor, can be coexpressed with HER2 in cell lines contributing to trastuzumab resistance through sustained Akt activation. HER2-overexpressing breast cancer cells 
respond to trastuzumab with a rapid upregulation of c-Met receptor expression and activation protecting cells against trastuzumab [91]. Loss of c-Met function by RNA interference improves the response of these cell lines to trastuzumab. Met-amplification, and certain alternative signals from Met to HER3 have been found capable of inducing acquired resistance to the EGFR TKIs gefitinib and erlotinib in nonsmall cell lung cancer [92].

7.2.4. Hormone Receptor Signaling. A potential mechanism of resistance is through ER (it says estrogen receptor) pathway. The existence of a potential crosstalk between HER2 and this pathway has been widely described [93-95]. Up-regulation of the estrogen receptor after treatment with lapatinib (anti-HER2 mAb) suggests that the receptor might act as a redundant survival pathway [96].

Crosstalk between the steroid hormone receptors for ER and progesterone (PR) and the HER family appears to be a hallmark of breast cancer growth. Proietti et al. [97] highlighted signal transducer and activator of transcription 3 (Stat3) as a key protein activated by heregulin (HRG), a ligand of the HER receptors through co-opted ligandindependent PR function as a signaling molecule. Stat 3 activation was an absolute requirement in HRG-induced mammary tumor growth, and targeting Stat 3 effectively inhibited growth of breast cancer cells with activated HRG/HER2 and PR. A novel potential therapeutic intervention in PR- and HER2-positive breast tumors involving the specific blockage of PR signaling activity could be investigated.

Other hormones such as prolactin acting through Janus kinase activation can transactivate HER2, leading to cell proliferation even in the presence of trastuzumab [98].

7.2.5. Cyclin E Amplification/Overexpression. The clinical relevance of cyclin E amplification/overexpression detected in numerous HER2 breast cancer patients has been reported [76], resulting in a lower clinical benefit rate and progression-free survival after trastuzumab therapy. High cyclin E expression has been proposed as a marker of poor clinical outcome in breast cancer [99]. Furthermore, it has been recently shown that cyclin E levels decrease upon HER2 downregulation, suggesting that HER2 regulates cyclin E function [100]. The role of cyclin $\mathrm{E}$ in trastuzumab resistance suggests that treatment with CDK inhibitors is a probable approach for patients whose tumors display cyclin $\mathrm{E}$ amplification/overexpression.

7.2.6. Tumor Necrosis Factor $\alpha$ Transactivation. Rivas et al. [101] have shown that tumor necrosis $\alpha$ (TNF $\alpha)$ induces HER2 phosphorylation in breast cancer cells, which is mediated by $\mathrm{c}-\mathrm{Src}$ activation. Moreover, TNF $\alpha$ promoted HER2/HER3 heterocomplex formation, Akt activation, and $\mathrm{NF}-\kappa \mathrm{B}$ transcriptional activation. Inhibition of HER2 by addition of AG825, an EGFR/HER2-tyrosine kinase inhibitor, or knockdown of HER2 by RNA interference blocked TNF $\alpha$-induced NFkB activation and cell proliferation. However, trastuzumab could not inhibit TNF $\alpha$ ability to promote breast cancer growth. Interestingly, $\mathrm{TNF} \alpha$ is able to transactivate HER2 and use it as a mandatory downstream signaling molecule in the generation of mitogenic signals. As TNF $\alpha$ has been shown to be present in the tumor microenvironment of a significant proportion of human infiltrating breast cancers, it would have clinical implication in HER2-positive breast cancer treatment.

7.2.7. Erythropoietin Coexpression. Erythropoietin (EPO) has long been known to be an important hematopoietic cytokine that regulates the survival, proliferation and differentiation of the erythroid progenitor cells in the bone marrow [102]. EPO-induced cell signaling via interaction with specific tyrosine-phosphorylated regions within the activated receptor for erythropoietin (EpoR), leads to activation of downstream signaling pathways, such as the MEK/Erk and PI3K/Akt pathways [103]. These downstream signaling pathways activated by EPO via EpoR overlap and/or interact with those activated by HER2.

Recently, Liang et al. [104] demonstrated that the EpoR is coexpressed with HER2 in a significant percentage of human breast tumor specimens and breast cancer cell lines. Exposure of these dual-positive breast cancer cells to recombinant human erythropoietin ( $\mathrm{rHuEPO}$ ), activated cell signaling. Concurrent cell treatment with rHuEPO and trastuzumab reduced the cell responsiveness to trastuzumab both in vitro and in vivo. They identified Jak2-mediated activation of Src and inactivation of PTEN as underlying mechanisms through which rHuEPO antagonizes trastuzumab-induced therapeutic effects.

7.2.8. Contribution of Rac1 Activity. Rac1, a Ras-like small GTPase, has been implicated in the control of cell growth and morphology and is believed to be associated with breast cancer progression. Dokmanovic et al. [105] using SKBR3 cells have reported that resistance to trastuzumab was associated with increased Racl activity, leading to significant cytoskeleton disorganization. Redution of Rac1 activity with a specific inhibitor, restored trastuzumab-mediated endocytic downregulation of HER2 and reduced extracellular signaling regulate kinase activity in resistant clones. In addition, the role of Rho GTPases as a treatment target has been recently reviewed by Menna et al. [106]. These results highlight Rac1 as a potential therapeutic target for the treatment of trastuzumab-resistant breast cancer.

\section{Impaired Immune-Mediated Mechanisms}

Breast tumor cells isolated from in vitro resistance to trastuzumab remain sensitive to trastuzumab antitumor effects in vivo and to ADCC killing. Tumor cells resistant to trastuzumab in vitro, remain sensitive to its antitumor effect in vivo as well as to antibody dependant cellular cytotoxicity (ADCC) killing [107]. Gene polymorphism producing the phenotype expression of valine $(\mathrm{V})$ or phenylalanine (F) at amino acid 158 on the Fc $\gamma$ RIIIa modifies the affinity of IgG1 to the Fcy receptor [108]. Immune effector cells carrying the Fc $\gamma$ RIIIa V/V alleles mediate a stronger ADCC 
of trastuzumab than cells bearing the F allele [109]. In the clinic, the Fc $\gamma$ RIII $158 \mathrm{~V} / \mathrm{F}$ polymorphism interferes with the ability to generate in vitro ADCC responses during trastuzumab treatment, impairing the clinical response and progression-free survival of patients in the metastatic setting [110]. Further evidence on the importance of ADCC was provided by Barok et al. [111], who showed that trastuzumab can trigger ADCC and destroy trastuzumab-resistant HER2 positive cell lines in vitro and in vivo xenografts.

In summary, these observations point to ADCC as a major player in trastuzumab antitumoral effect. It would be very interesting to know how ADCC affects cells that are prone to enhance PI3K/Akt and RAS/Raf/MAPK pathways in order to survive. Multiple proteases involved with infection, inflammation, and tumor environments are able to cleave IgG molecules on site, generating monovalent or bivalent antibody fragments, Fab' or F( $\left.\mathrm{ab}^{\prime}\right)$, which lack Fc-mediated effector function [112]. However, these antibody fragments can still bind to their cognate antigen, but lack binding sites for complement and for Fc receptors, and can no longer trigger Fc-mediated functions such as ADCC.

In our laboratory, we are studying the mechanisms involved in trastuzumab effect and resistance in different human breast cancer cells overexpressing HER2. Our study is mainy focused using 3D cultures of tumor cells (spheroids) in presence or not of syngeneic macrophages or peripheral mononuclear cells $[113,114]$.

\section{Conclusion}

Trastuzumab is an example of targeted therapy that offers significant clinical benefit in patients with HER2 overexpressing cancers in the adjuvant and metastatic settings. However, nearly $70 \%$ of patients, who initially reasonable option trastuzumab, demonstrate disease progression within 1 year of treatment. Elucidation of the mechanisms involved in de novo and acquired resistance to trastuzumab seem to be difficult due to the multiple mechanisms of action. A strategy to overcome resistance may involve the use of treatments that do not depend on molecular binding to the HER2 extracellular domain, including the use of TK inhibitors such as lapatinib or of inhibitors of the downstream HER2 signaling pathways. Another reasonable option would be to increase tumor cell destruction through extracellular mechanisms by increasing or enabling ADCC or by using immunotoxins such as the antibody-drug conjugate trastuzumab-DM1 [115] or the bispecific anti-HER2/CD3 Ab ertumaxomab.

Based on emerging knowledge of the HER2-signaling pathways and of signaling through alternative pathways, several drugs against HER2-overexpressing breast cancers are in different phases of clinical development, whereas others are still struggling to leave the bench.

It will be of great clinical impact to discover and identify potential molecular predictors of response to current and new HER2-targeted agents. Understanding the mechanisms by which HER2 can be activated by nonclassical receptors and ligands is relevant in order not only to design new therapeutic approaches, but also to predict patient's response.
Many new agents are currently in preclinical stages of development. They include HER2 targeting agents as mAbs, TKIs, and vaccines. Pertuzumab, a recombinant humanized HER mAb that blocks dimerization of HER2 with HER3 and EGFR [116], is one of the new classes of drugs called dimerization inhibitors. It has the potential to block signaling by other HER family receptors as well as inhibiting signaling in cells that express normal levels of HER.

\section{Acknowledgments}

The authors thank University of Buenos Aires (Grants UBACYT M415 and 01/2366) and to the National Agency for Scientific and Technical Promotion (Grant PICT 417).

\section{References}

[1] J. Ferlay, H. R. Shin, F. Bray, D. Forman, C. Mathers, and D. M. Parkin, "Cancer Incidence and Mortality Worldwide: IARC," Globocan 2008, IARC CancerBase http://globocan. iarc.fr .

[2] T. Sørlie, C. M. Perou, R. Tibshirani et al., "Gene expression patterns of breast carcinomas distinguish tumor subclasses with clinical implications," Proceedings of the National Academy of Sciences of the United States of America, vol. 98, no. 19, pp. 10869-10874, 2001.

[3] D. J. Slamon, B. Leyland-Jones, S. Shak et al., "Use of chemotherapy plus a monoclonal antibody against her2 for metastatic breast cancer that overexpresses HER2," New England Journal of Medicine, vol. 344, no. 11, pp. 783-792, 2001.

[4] Z. Hu, C. Fan, D. S. Oh et al., "The molecular portraits of breast tumors are conserved across microarray platforms," BMC Genomics, vol. 7, article no. 96, 2006.

[5] B. P. Zhou, M. C. T. Hu, S. A. Miller et al., "HER-2/neu blocks tumor necrosis factor-induced apoptosis via the Akt/NF- $\kappa \mathrm{B}$ pathway," Journal of Biological Chemistry, vol. 275, no. 11, pp. 8027-8031, 2000.

[6] M. A. Olayioye, R. M. Neve, H. A. Lane, and N. E. Hynes, "The ErbB signaling network: receptor heterodimerization in development and cancer," EMBO Journal, vol. 19, no. 13, pp. 3159-3167, 2000.

[7] P. R. Pohlmann, I. A. Mayer, and R. Mernaugh, "Resistance to trastuzumab in breast cancer," Clinical Cancer Research, vol. 15, no. 24, pp. 7479-7491, 2009.

[8] H. A. Lane, I. Beuvink, A. B. Motoyama, J. M. Daly, R. M. Neve, and N. E. Hynes, "ErbB2 potentiates breast tumor proliferation through modulation of p27(Kip1)-Cdk2 complex formation: receptor overexpression does not determine growth dependency," Molecular and Cellular Biology, vol. 20, no. 9, pp. 3210-3223, 2000.

[9] J. Schlessinger, "Common and distinct elements in cellular signaling via EGF and FGF receptor," Science, vol. 306, no. 5701, pp. 1506-1507, 2004.

[10] N. E. Hynes and H. A. Lane, "ERBB receptors and cancer: the complexity of targeted inhibitors," Nature Reviews Cancer, vol. 5, no. 5, pp. 341-354, 2005.

[11] D. S. Salomon, R. Brandt, F. Ciardiello, and N. Normanno, "Epidermal growth factor-related peptides and their receptors in human malignancies," Critical Reviews in Oncology/ Hematology, vol. 19, no. 3, pp. 183-232, 1995.

[12] D. J. Slamon, G. M. Clark, S. G. Wong, W. J. Levin, A. Ullrich, and W. L. McGuire, "Human breast cancer: correlation of 
relapse and survival with amplification of the HER-2/neu oncogene," Science, vol. 235, no. 4785, pp. 182-191, 1987.

[13] J. Jaehne, C. Urmacher, H. T. Thaler, H. Friedlander-Klar, C. Cordon-Cardo, and H. J. Meyer, "Expression of Her2/neu oncogene product p185 in correlation to clinicopathological and prognostic factors of gastric carcinoma," Journal of Cancer Research and Clinical Oncology, vol. 118, no. 6, pp. 474479, 1992.

[14] J. Vermeij, E. Teugels, C. Bourgain et al., "Genomic activation of the EGFR and HER2-neu genes in a significant proportion of invasive epithelial ovarian cancers," BMC Cancer, vol. 8, article no. 3, 2008.

[15] G. Cornolti, M. Ungari, M. L. Morassi et al., "Amplification and overexpression of Her2/neu gene and HER2/neu protein in salivary duct carcinoma of the parotid gland," Archives of Otolaryngology - Head and Neck Surgery, vol. 133, no. 10, pp. 1031-1036, 2007.

[16] R. M. Hudziak, J. Schlessinger, and A. Ullrich, "Increased expression of the putative growth factor receptor p185HER2 causes transformation and tumorigenesis of NIH 3T3 cells," Proceedings of the National Academy of Sciences of the United States of America, vol. 84, no. 20, pp. 7159-7163, 1987.

[17] A. Choudhury and R. Kiessling, "Her-2/Neu as a paradigm of a tumor-specific target for therapy," Breast Disease, vol. 20, pp. 25-31, 2004.

[18] D. S. Leonard, A. D. K. Hill, L. Kelly, B. Dijkstra, E. McDermott, and N. J. O'Higgins, "Anti-human epidermal growth factor receptor 2 monoclonal antibody therapy for breast cancer," British Journal of Surgery, vol. 89, no. 3, pp. 262-271, 2002.

[19] M. F. Press, D. J. Slamon, K. J. Flom, J. Park, J. Y. Zhou, and L. Bernstein, "Evaluation of HER-2/neu gene amplification and overexpression: comparison of frequently used assay methods in a molecularly characterized cohort of breast can-cer specimens," Journal of Clinical Oncology, vol. 20, no. 14, pp. 3095-3105, 2002.

[20] J. Baselga and S. M. Swain, "Novel anticancer targets: revisiting ERBB2 and discovering ERBB3," Nature Reviews Cancer, vol. 9, no. 7, pp. 463-475, 2009.

[21] A. B. Motoyama, N. E. Hynes, and H. A. Lane, "The efficacy of ErbB receptor-targeted anticancer therapeutics is influenced by the availability of epidermal growth factor-related peptides," Cancer Research, vol. 62, no. 11, pp. 31513158, 2002.

[22] A. M. V. Petit, J. Rak, M. C. Hung et al., "Neutralizing antibodies against epidermal growth factor and ErbB-2/neu receptor tyrosine kinases down-regulate vascular endothelial growth factor production by tumor cells in vitro and in vivo: angiogenic implications for signal transduction therapy of solid tumors," American Journal of Pathology, vol. 151, no. 6, pp. 1523-1530, 1997.

[23] P. Carter, L. Presta, C. M. Gorman et al., "Humanization of an anti-p185(HER2) antibody for human cancer therapy," Proceedings of the National Academy of Sciences of the United States of America, vol. 89, no. 10, pp. 4285-4289, 1992.

[24] A. D. Seidman, D. Berry, C. Cirrincione et al., "Randomized phase III trial of weekly compared with every-3-weeks paclitaxel for metastatic breast cancer, with trastuzumab for all HER-2 overexpressors and random assignment to trastuzumab or not in HER-2 nonoverexpressors: final results of cancer and leukemia group B protocol 9840," Journal of Clinical Oncology, vol. 26, no. 10, pp. 1642-1649, 2008.
[25] R. Nahta and F. J. Esteva, "HER2 therapy: molecular mechanisms of trastuzumab resistance," Breast Cancer Research, vol. 8, no. 6, article no. 215, 2006.

[26] C. L. Vogel, M. A. Cobleigh, D. Tripathy et al., "Efficacy and safety of trastuzumab as a single agent in first-line treatment of HER2-overexpressing metastatic breast cancer," Journal of Clinical Oncology, vol. 20, no. 3, pp. 719-726, 2002.

[27] M. A. Cobleigh, C. L. Vogel, D. Tripathy et al., "Multinational study of the efficacy and safety of humanized antiHER2 monoclonal antibody in women who have HER2overexpressing metastatic breast cancer that has progressed after chemotherapy for metastatic disease," Journal of Clinical Oncology, vol. 17, no. 9, pp. 2639-2648, 1999.

[28] E. H. Romond, E. A. Perez, J. Bryant et al., “Trastuzumab plus adjuvant chemotherapy for operable HER2-positive breast cancer," New England Journal of Medicine, vol. 353, no. 16, pp. 1673-1684, 2005.

[29] E. A. Perez, M. M. Reinholz, D. W. Hillman et al., "HER2 and chromosome 17 effect on patient outcome in the N9831 adjuvant trastuzumab trial," Journal of Clinical Oncology, vol. 28, no. 28, pp. 4307-4315, 2010.

[30] G. Valabrega, F. Montemurro, and M. Aglietta, "Trastuzumab: mechanism of action, resistance and future perspectives in HER2-overexpressing breast cancer," Annals of Oncology, vol. 18, no. 6, pp. 977-984, 2007.

[31] J. Baselga, J. Albanell, M. A. Molina, and J. Arribas, "Mechanism of action of trastuzumab and scientific update," Seminars in Oncology, vol. 28, no. 5, pp. 4-11, 2001.

[32] H. A. Lane, A. B. Motoyama, I. Beuvink, and N. E. Hynes, "Modulation of p27/Cdk2 complex formation through 4D5mediated inhibition of HER2 receptor signaling," Annals of Oncology, vol. 12, no. 1, pp. S21-S22, 2001.

[33] Y. Nagata, K. H. Lan, X. Zhou et al., "PTEN activation contributes to tumor inhibition by trastuzumab, and loss of PTEN predicts trastuzumab resistance in patients," Cancer Cell, vol. 6, no. 2, pp. 117-127, 2004.

[34] T. A. Christianson, J. K. Doherty, Y. J. Lin et al., "NH2terminally truncated HER-2/neu protein: relationship with shedding of the extracellular domain and with prognostic factors in breast cancer," Cancer Research, vol. 58, no. 22, pp. 5123-5129, 1998.

[35] M. A. Molina, J. Codony-Servat, J. Albanell, F. Rojo, J. Arribas, and J. Baselga, "Trastuzumab (Herceptin), a humanized anti-HER2 receptor monoclonal antibody, inhibits basal and activated HER2 ectodomain cleavage in breast cancer cells," Cancer Research, vol. 61, no. 12, pp. 4744-4749, 2001.

[36] M. N. Fornier, A. D. Seidman, M. K. Schwartz et al., "Serum HER2 extracellular domain in metastatic breast cancer patients treated with weekly trastuzumab and paclitaxel: association with HER2 status by immunohistochemistry and fluorescence in situ hybridization and with response rate," Annals of Oncology, vol. 16, no. 2, pp. 234-239, 2005.

[37] Y. Izumi, L. Xu, E. Di Tomaso, D. Fukumura, and R. K. Jain, "Herceptin acts as an anti-angiogenic cocktail," Nature, vol. 416, no. 6878, pp. 279-280, 2002.

[38] X. F. Wen, G. Yang, W. Mao et al., "HER2 signaling modulates the equilibrium between pro- and antiangiogenic factors via distinct pathways: implications for HER2-targeted antibody therapy," Oncogene, vol. 25, no. 52, pp. 6986-6996, 2006.

[39] A. M. Hommelgaard, M. Lerdrup, and B. Van Deurs, "Association with Membrane Protrusions Makes ErbB2 an Internalization-resistant Receptor," Molecular Biology of the Cell, vol. 15, no. 4, pp. 1557-1567, 2004.

[40] M. Cuello, S. A. Ettenberg, A. S. Clark et al., "Down-regulation of the erbB-2 receptor by trastuzumab (Herceptin) 
enhances tumor necrosis factor-related apoptosis-inducing ligand-mediated apoptosis in breast and ovarian cancer cell lines that overexpress erbB-2," Cancer Research, vol. 61, no. 12, pp. 4892-4900, 2001.

[41] T. Ben-Kasus, B. Schechter, S. Lavi, Y. Yarden, and M. Sela, "Persistent elimination of ErbB-2/HER2-overexpressing tu mors using combinations of monoclonal antibodies: relevance of receptor endocytosis," Proceedings of the National Academy of Sciences of the United States of America, vol. 106, no. 9, pp. 3294-3299, 2009.

[42] C. C. Portera, J. M. Walshe, D. R. Rosing et al., "Cardiac toxicity and efficacy of trastuzumab combined with pertuzumab in patients with trastuzumab-insensitive human epidermal growth factor receptor 2- Positive metastatic breast cancer," Clinical Cancer Research, vol. 14, no. 9, pp. 2710-2716, 2008.

[43] C. I. Spiridon, S. Guinn, and E. S. Vitetta, "A comparison of the in vitro and in vivo activities of $\operatorname{IgG}$ and $\mathrm{F}(\mathrm{ab}$ ') 2 fragments of a mixture of three monoclonal anti-Her-2 antibodies," Clinical Cancer Research, vol. 10, no. 10, pp. 35423551, 2004.

[44] B. Stockmeyer, T. Beyer, W. Neuhuber et al., "Polymorphonuclear Granulocytes Induce Antibody-Dependent Apoptosis in Human Breast Cancer Cells," Journal of Immunology, vol. 171, no. 10, pp. 5124-5129, 2003.

[45] R. Gennari, S. Menard, F. Fagnoni et al., "Pilot study of the mechanism of action of preoperative trastuzumab in patients with primary operable breast tumors overexpressing HER2," Clinical Cancer Research, vol. 10, no. 17, pp. 5650-5655, 2004.

[46] R. J. Pietras, B. M. Fendly, V. R. Chazin, M. D. Pegram, S. B. Howell, and D. J. Slamon, "Antibody to HER-2/neu recep-tor blocks DNA repair after cisplatin in human breast and ovarian cancer cells," Oncogene, vol. 9, no. 7, pp. 1829-1838, 1994.

[47] R. J. Pietras, J. C. Poen, D. Gallardo, P. N. Wongvipat, J. J. Lee, and D. J. Slamon, "Monoclonal antibody to HER-2/neu receptor modulates repair of radiation- induced DNA damage and enhances radiosensitivity of human breast can-cer cells overexpressing this oncogene," Cancer Research, vol. 59, no. 6, pp. 1347-1355, 1999.

[48] R. Nahta, D. Yu, M. C. Hung, G. N. Hortobagyi, and F. J. Esteva, "Mechanisms of disease: understanding resistance to HER2-targeted therapy in human breast cancer," Nature Clinical Practice Oncology, vol. 3, no. 5, pp. 269-280, 2006.

[49] M. A. Molina, R. Sáez, E. E. Ramsey et al., "NH2-terminal truncated HER-2 protein but not full-length receptor is associated with nodal metastasis in human breast cancer," Clinical Cancer Research, vol. 8, no. 2, pp. 347-353, 2002.

[50] M. Scaltriti, F. Rojo, A. Ocaña et al., "Expression of p95HER2, a truncated form of the HER2 receptor, and response to AntiHER2 therapies in breast cancer," Journal of the National Cancer Institute, vol. 99, no. 8, pp. 628-638, 2007.

[51] S. Lennon, C. Barton, L. Banken, L. Gianni, M. Marty, and J. Baselga, "Utility of serum HER2 extracellular domain assessment in clinical decision making: pooled analysis of four trials of trastuzumab in metastatic breast cancer," Jour-nal of Clinical Oncology, vol. 27, no. 10, pp. 1685-1693, 2009.

[52] K. L. Carraway and N. Idris, "Regulation of sialomucin complex/Muc4 in the female rat reproductive tract," Biochemical Society Transactions, vol. 29, no. 2, pp. 162-166, 2001.

[53] K. L. Carraway, S. A. Price-Schiavi, M. Komatsu, S. Jepson, A. Perez, and C. A. Carothers Carraway, "Muc4/sialomucin complex in the mammary gland and breast cancer," Journal of Mammary Gland Biology and Neoplasia, vol. 6, no. 3, pp. 323-337, 2001.

[54] S. Mahanta, S. P. Fessles, J. Park, and C. Bamdad, "A minimal fragments of MUC1 mediates growth of cancer cells," PLoS ONE, vol. 3, no. 4, Article ID e2054, 2008.
[55] S. A. Price-Schiavi, S. Jepson, P. Li et al., "Rat MUC4 (sialomucin complex) reduces binding of anti-ErbB2 antibodies to tumor cell surfaces, a potential mechanism for herceptin resistance," International Journal of Cancer, vol. 99, no. 6, pp. 783-791, 2002.

[56] P. Nagy, E. Friedländer, M. Tanner et al., "Decreased accessibility and lack of activation of ErbB2 in JIMT-1, a herceptin-resistant, MUC4-expressing cancer cell line," Cancer Research, vol. 65, no. 2, pp. 473-482, 2005.

[57] L. Y. W. Bourguignon, H. Zhu, B. Zhou, F. Diedrich, P. A. Singleton, and M. C. Hung, "Hyaluronan Promotes CD44v3Vav2 Interaction with Grb2-p185HER2 and Induces Rac1 and Ras Signaling during Ovarian Tumor Cell Migration and Growth," Journal of Biological Chemistry, vol. 276, no. 52, pp. 48679-48692, 2001.

[58] S. Ghatak, S. Misra, and B. P. Toole, "Hyaluronan oligosaccharides inhibit anchorage-independent growth of tumor cells by suppressing the phosphoinositide 3-kinase/Akt cell survival pathway," Journal of Biological Chemistry, vol. 277, no. 41, pp. 38013-38020, 2002.

[59] P. P. Pandolfi, "Breast cancer - Loss of PTEN predicts resistance to treatment," New England Journal of Medicine, vol. 351, no. 22, pp. 2337-2338, 2004.

[60] L. Simpson and R. Parsons, "PTEN: life as a tumor suppressor," Experimental Cell Research, vol. 264, no. 1, pp. 29-41, 2001.

[61] F. M. Yakes, W. Chinratanalab, C. A. Ritter, W. King, S. Seelig, and C. L. Arteaga, "Herceptin-induced inhibition of phosphatidylinositol-3 kinase and Akt is required for antibodymediated effects on p27, cyclin D1, and antitumor action," Cancer Research, vol. 62, no. 14, pp. 4132-4141, 2002.

[62] K. Berns, H. M. Horlings, B. T. Hennessy et al., "A functional genetic approach identifies the PI3K pathway as a major determinant of trastuzumab resistance in breast cancer," Cancer Cell, vol. 12, no. 4, pp. 395-402, 2007.

[63] B. Dave, I. Migliaccio, M. C. Gutierrez et al., "Loss of phosphatase and tensin homolog or phosphoinositol-3 kinase activation and response to trastuzumab or lapatinib in human epidermal growth factor receptor 2 - Overexpressing locally advanced breast cancers," Journal of Clinical Oncology, vol. 29, no. 2, pp. 166-173, 2011.

[64] C. Jimenez, D. R. Jones, P. Rodríguez-Viciana et al., "Identification and characterization of a new oncogene derived from the regulatory subunit of phosphoinositide 3-kinase," EMBO Journal, vol. 17, no. 3, pp. 743-753, 1998.

[65] A. J. Philp, I. G. Campbell, C. Leet et al., "The phosphatidylinositol 3'-kinase p85 $\alpha$ gene is an oncogene in human ovarian and colon tumors," Cancer Research, vol. 61, no. 20, pp. 74267429, 2001.

[66] L. H. Saal, K. Holm, M. Maurer et al., "PIK3CA mutations correlate with hormone receptors, node metastasis, and ERBB2, and are mutually exclusive with PTEN loss in human breast carcinoma," Cancer Research, vol. 65, no. 7, pp. 25542559, 2005.

[67] T. T. Junttila, R. W. Akita, K. Parsons et al., "LigandIndependent HER2/HER3/PI3K Complex Is Disrupted by Trastuzumab and Is Effectively Inhibited by the PI3K Inhibitor GDC-0941," Cancer Cell, vol. 15, no. 5, pp. 429-440, 2009.

[68] Y. Kataoka, T. Mukohara, H. Shimada, N. Saijo, M. Hirai, and H. Minami, "Association between gain-of-function mutations in PIK3CA and resistance to HER2-targeted agents in HER2-amplified breast cancer cell lines," Annals of Oncology, vol. 21, no. 2, pp. 255-262, 2010. 
[69] J. Dunlap, C. Le, A. Shukla et al., "Phosphatidylinositol-3kinase and AKT1 mutations occur early in breast carcinoma," Breast Cancer Research and Treatment, vol. 120, no. 2, pp. 409-418, 2010.

[70] K. Stemke-Hale, A. M. Gonzalez-Angulo, A. Lluch et al., "An integrative genomic and proteomic analysis of PIK3CA, PTEN, and AKT mutations in breast cancer," Cancer Research, vol. 68, no. 15, pp. 6084-6091, 2008.

[71] J. Brugge, M. C. Hung, and G. B. Mills, "A New Mutational aktivation in the PI3K Pathway," Cancer Cell, vol. 12, no. 2, pp. 104-107, 2007.

[72] P. H. Tseng, Y. C. Wang, S. C. Weng et al., "Overcoming trastuzumab resistance in HER2-overexpressing breast cancer cells by using a novel celecoxib-derived phosphoinositide-dependent kinase-1 inhibitor," Molecular Pharmacology, vol. 70, no. 5, pp. 1534-1541, 2006.

[73] R. M. Neve, H. Sutterlüty, N. Pullen et al., "Effects of oncogenic ErbB2 on G1 cell cycle regulators in breast tumour cells," Oncogene, vol. 19, no. 13, pp. 1647-1656, 2000.

[74] R. Nahta, T. Takahashi, N. T. Ueno, M. C. Hung, and F. J. Esteva, "P27kip1 down-regulation is associated with trastuzumab resistance in breast cancer cells," Cancer Research, vol. 64, no. 11, pp. 3981-3986, 2004.

[75] X. F. Le, F. X. Claret, A. Lammayot et al., "The role of cyclindependent kinase inhibitor p27Kip1 in anti-HER2 antibodyinduced G1 cell cycle arrest and tumor growth inhibition," Journal of Biological Chemistry, vol. 278, no. 26, pp. 2344123450, 2003.

[76] M. Scaltriti, P. J. Eichhorn, J. Cortés et al., "Cyclin E amplification/overexpression is a mechanism of trastuzumab resistance in HER2+ breast cancer patients," Proceedings of the National Academy of Sciences of the United States of America, vol. 108, no. 9, pp. 3761-3766, 2011.

[77] X. F. Le, F. Pruefer, and R. C. Bast, "HER2-targeting antibodies modulate the cyclin-dependent kinase inhibitor p27Kip1 via multiple signaling pathways," Cell Cycle, vol. 4, no. 1, pp. 87-95, 2005.

[78] S. Diermeier, G. Horváth, R. Knuechel-Clarke, F. Hofstaedter, J. Szöllosi, and G. Brockhoff, "Epidermal growth factor receptor coexpression modulates susceptibility to Herceptin in HER2/neu overexpressing breast cancer cells via specific erbB-receptor interaction and activation," Experimental Cell Research, vol. 304, no. 2, pp. 604-619, 2005.

[79] G. Brockhoff, B. Heckel, E. Schmidt-Bruecken et al., "Differential impact of Cetuximab, Pertuzumab and Trastuzumab on BT474 and SK-BR-3 breast cancer cell proliferation," Cell Proliferation, vol. 40, no. 4, pp. 488-507, 2007.

[80] L. M. Bender and R. Nahta, "Her2 cross talk and therapeutic resistance in breast cancer," Frontiers in Bioscience, vol. 13, no. 10, pp. 3906-3912, 2008.

[81] E. Tzahar, H. Waterman, X. Chen et al., "A hierarchical network of interreceptor interactions determines signal transduction by Neu differentiation factor/neuregulin and epidermal growth factor," Molecular and Cellular Biology, vol. 16, no. 10, pp. 5276-5287, 1996.

[82] S. T. Lee-Hoeflich, L. Crocker, E. Yao et al., "A central role for HER3 in HER2-amplified breast cancer: implications for targeted therapy," Cancer Research, vol. 68, no. 14, pp. 58785887, 2008.

[83] T. Holbro, R. R. Beerli, F. Maurer, M. Koziczak, C. F. Barbas, and N. E. Hynes, "The ErbB2/ErbB3 heterodimer functions as an oncogenic unit: erbB2 requires ErbB3 to drive breast tumor cell proliferation," Proceedings of the National Academy of Sciences of the United States of America, vol. 100, no. 15, pp. 8933-8938, 2003.

[84] T. S. Wehrman, W. J. Raab, C. L. Casipit, R. Doyonnas, J. H. Pomerantz, and H. M. Blau, "A system for quantifying dynamic protein interactions defines a role for Herceptin in modulating ErbB2 interactions," Proceedings of the National Academy of Sciences of the United States of America, vol. 103, no. 50, pp. 19063-19068, 2006.

[85] G. Valabrega, F. Montemurro, I. Sarotto et al., "TGF $\alpha$ expression impairs Trastuzumab-induced HER2 downregulation," Oncogene, vol. 24, no. 18, pp. 3002-3010, 2005.

[86] A. G. Robinson, D. Turbin, T. Thomson et al., "Molecular predictive factors in patients receiving trastuzumab-based chemotherapy for metastatic disease," Clinical Breast Cancer, vol. 7, no. 3, pp. 254-261, 2006.

[87] Y. Lu, X. Zi, Y. Zhao, D. Mascarenhas, and M. Pollak, "Insulin-like growth factor-I receptor signaling and resistance to transtuzumab (Herceptin)," Journal of the National Cancer Institute, vol. 93, no. 24, pp. 1852-1857, 2001.

[88] R. Nahta, L. X. H. Yuan, B. Zhang, R. Kobayashi, and F. J. Esteva, "Insulin-like growth factor-I receptor/human epidermal growth factor receptor 2 heterodimerization contributes to trastuzumab resistance of breast cancer cells," Cancer Research, vol. 65, no. 23, pp. 11118-11128, 2005.

[89] Y. Lu, X. Zi, and M. Pollak, "Molecular mechanisms underlying IGF-I-induced attenuation of the growth-inhibitory activity of trastuzumab (herceptin) on SKBR3 breast cancer cells," International Journal of Cancer, vol. 108, no. 3, pp. 334341, 2004.

[90] L. Yen, X. L. You, A. E. Al Moustafa et al., "Heregulin selectively upregulates vascular endothelial growth factor secretion in cancer cells and stimulates angiogenesis," Oncogene, vol. 19, no. 31, pp. 3460-3469, 2000.

[91] D. L. Shattuck, J. K. Miller, K. L. Carraway, and C. Sweeney, "Met receptor contributes to trastuzumab resistance of Her2overexpressing breast cancer cells," Cancer Research, vol. 68, no. 5, pp. 1471-1477, 2008.

[92] J. A. Engelman, K. Zejnullahu, T. Mitsudomi et al., "MET amplification leads to gefitinib resistance in lung cancer by activating ERBB3 signaling," Science, vol. 316, no. 5827, pp. 1039-1043, 2007.

[93] A. Jones, "Combining trastuzumab (Herceptinß) with hormonal therapy in breast cancer: what can be expected and why?" Annals of Oncology, vol. 14, no. 12, pp. 1697-1704, 2003.

[94] S. R. D. Johnston and M. Dowsett, "Aromatase inhibitors for breast cancer: lessons from the laboratory," Nature Reviews Cancer, vol. 3, no. 11, pp. 821-831, 2003.

[95] A. Ocaña, J. J. Cruz, and A. Pandiella, "Trastuzumab and antiestrogen therapy: focus on mechanisms of action and resistance," American Journal of Clinical Oncology: Cancer Clinical Trials, vol. 29, no. 1, pp. 90-95, 2006.

[96] W. Xia, S. Bacus, P. Hegde et al., "A model of acquired autoresistance to a potent ErbB2 tyrosine kinase inhibitor and a therapeutic strategy to prevent its onset in breast cancer," Proceedings of the National Academy of Sciences of the United States of America, vol. 103, no. 20, pp. 7795-7800, 2006.

[97] C. J. Proietti, C. Rosemblit, W. Beguelin et al., "Activation of Stat3 by heregulin/ErbB-2 through the co-option of progesterone receptor signaling drives breast cancer growth," Molecular and Cellular Biology, vol. 29, no. 5, pp. 1249-1265, 2009.

[98] T. Yamauchi, N. Yamauchi, K. Ueki et al., "Constitutive tyrosine phosphorylation of ErbB-2 via Jak2 by autocrine 
secretion of prolactin in human breast cancer," Journal of Biological Chemistry, vol. 275, no. 43, pp. 33937-33944, 2000.

[99] K. Keyomarsi, S. L. Tucker, T. A. Buchholz et al., "Cyclin E and survival in patients with breast cancer," New England Journal of Medicine, vol. 347, no. 20, pp. 1566-1575, 2002.

[100] E. A. Mittendorf, Y. Liu, S. L. Tucker et al., "A novel interaction between HER2/neu and cyclin e in breast cancer," Oncogene, vol. 29, no. 27, pp. 3896-3907, 2010.

[101] M. A. Rivas, M. Tkach, W. Beguelin et al., "Transactivation of ErbB-2 induced by tumor necrosis factor $\alpha$ promotes NF- $\kappa$ B activation and breast cancer cell proliferation," Breast Cancer Research and Treatment, vol. 122, no. 1, pp. 111-124, 2010.

[102] W. Jelkmann, "Erythropoietin: structure, control of production, and function," Physiological Reviews, vol. 72, no. 2, pp. 449-489, 1992.

[103] J. E. Damen, L. Liu, R. L. Cutler, and G. Krystal, "Erythropoietin stimulates the tyrosine phosphorylation of Shc and its association with Grb2 and a 145-Kd tyrosine phosphorylated protein," Blood, vol. 82, no. 8, pp. 2296-2303, 1993.

[104] K. Liang, F. J. Esteva, C. Albarracin et al., "Recombinant human erythropoietin antagonizes trastuzumab treatment of breast cancer cells via Jak2-Mediated Src activation and PTEN inactivation," Cancer Cell, vol. 18, no. 5, pp. 423-435, 2010.

[105] M. Dokmanovic, D. S. Hirsch, Y. Shen, and J. W. Wen, "Rac1 contributes to trastuzumab resistance of breast cancer cells: Rac1 as a potential therapeutic target for the treatment of trastuzumab-resistant breast cancer," Molecular Cancer Therapeutics, vol. 8, no. 6, pp. 1557-1569, 2009.

[106] P. L. Menna, G. A. Cardama, M. J. Comin, D. F. Alonso, and D. E. Gomez, "Rho GTPases as therapeutic targets in cancer and other human diseases," Medicina, vol. 70, no. 6, pp. 555$564,2010$.

[107] T. E. Kute, L. Savage, J. R. Stehle Jr. et al., "Breast tumor cells isolated from in vitro resistance to trastuzumab remain sensitive to trastuzumab anti-tumor effects in vivo and to ADCC killing," Cancer immunology, immunotherapy, vol. 58, no. 11, pp. 1887-1896, 2009.

[108] H. R. Koene, M. Kleijer, J. Algra, D. Roos, A. E. G. K. Von Dem Borne, and M. De Haas, "Fc $\gamma$ RIIIa-158V/F polymorphism influences the binding of IgG by natural killer cell FC $\gamma$ RIIIa, independently of the FC $\gamma$ RIIIa-48L/R/H phenotype," Blood, vol. 90, no. 3, pp. 1109-1114, 1997.

[109] R. L. Shields, A. K. Namenuk, K. Hong et al., "High Resolution Mapping of the Binding Site on Human IgG1 for Fc $\gamma$ RI, Fc $\gamma$ RII, Fc $\gamma$ RIII, and FcRn and Design of IgG1 Variants with Improved Binding to the Fc $\gamma \mathrm{R}$," Journal of Biological Chemistry, vol. 276, no. 9, pp. 6591-6604, 2001.

[110] A. Musolino, N. Naldi, B. Bortesi et al., "Immunoglobulin $\mathrm{g}$ fragment $\mathrm{c}$ receptor polymorphisms and clinical efficacy of trastuzumab-based therapy in patients with HER-2/neupositive metastatic breast cancer," Journal of Clinical Oncology, vol. 26, no. 11, pp. 1789-1796, 2008.

[111] M. Barok, J. Isola, Z. Pályi-Krekk et al., "Trastuzumab causes antibody-dependent cellular cytotoxicity-mediated growth inhibition of submacroscopic JIMT-1 breast cancer xenografts despite intrinsic drug resistance," Molecular Cancer Therapeutics, vol. 6, no. 7, pp. 2065-2072, 2007.

[112] A. J. H. Gearing, S. J. Thorpe, K. Miller et al., "Selective cleavage of human IgG by the matrix metalloproteinases, matrilysin and stromelysin," Immunology Letters, vol. 81, no. 1, pp. 41-48, 2002.

[113] J. S. Roldán, A. C. Najenson, M. F. Roca, L. Marino, M. A. Jasnis, and G. L. Fiszman, "Study of the immune system participation in tumor progression using a $3 \mathrm{D}$ culture model of adenocarcinoma cells and macrophages," Medicina (Buenos Aires), vol. 69, article 174, 2009.

[114] J. S. Roldán, A. C. Najenson, M. F. Roca, L. Marino, M. A. Jasnis, and G. L. Fiszman, "Interaction between mammary adenocarcinoma cells and macrophages in a three-dimensional model of tumor spheroids," in Proceedings of the AACR, Washington, DC, USA, 2010, Abstract \#548.

[115] M. Barok, M. Tanner, K. Koninki, and J. Isola, "TrastuzumabDM1 causes tumor growth inhibition by mitotic catastrophe in trastuzumab-resistant breast cancer cells in vivo," Breast Cancer Research, p. R46, 2011.

[116] L. Gianni, A. Lladó, G. Bianchi et al., "Open-label, phase II, multicenter, randomized study of the efficacy and safety of two dose levels of pertuzumab, a human epidermal growth factor receptor 2 dimerization inhibitor, in patients with human epidermal growth factor receptor 2-negative meta-static breast cancer," Journal of Clinical Oncology, vol. 28, no. 7, pp. 1131-1137, 2010. 


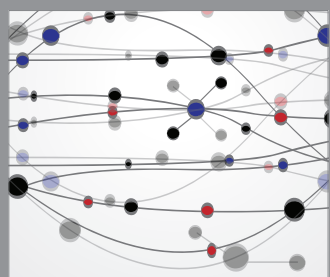

The Scientific World Journal
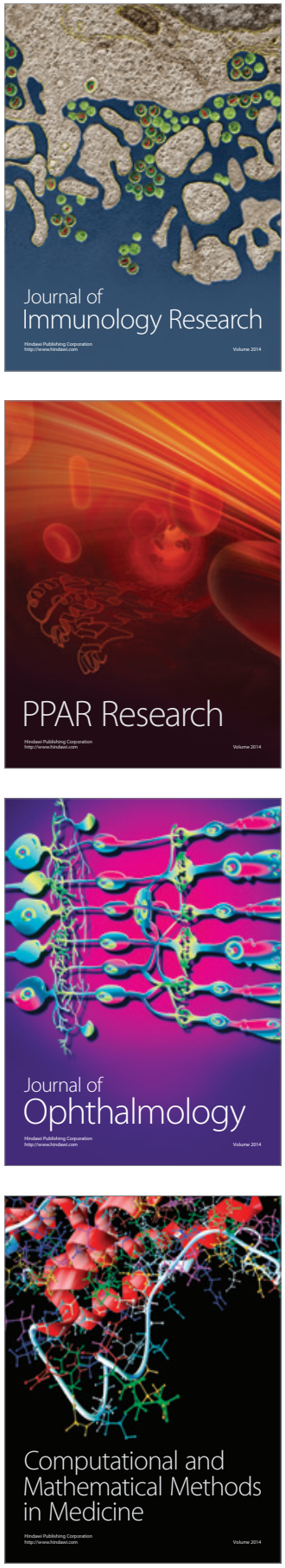

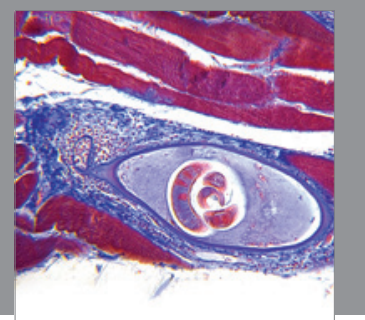

Gastroenterology

Research and Practice
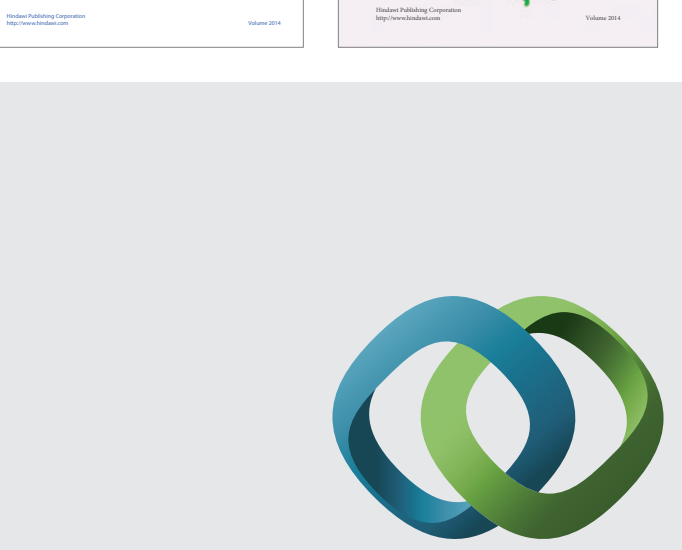

\section{Hindawi}

Submit your manuscripts at

http://www.hindawi.com
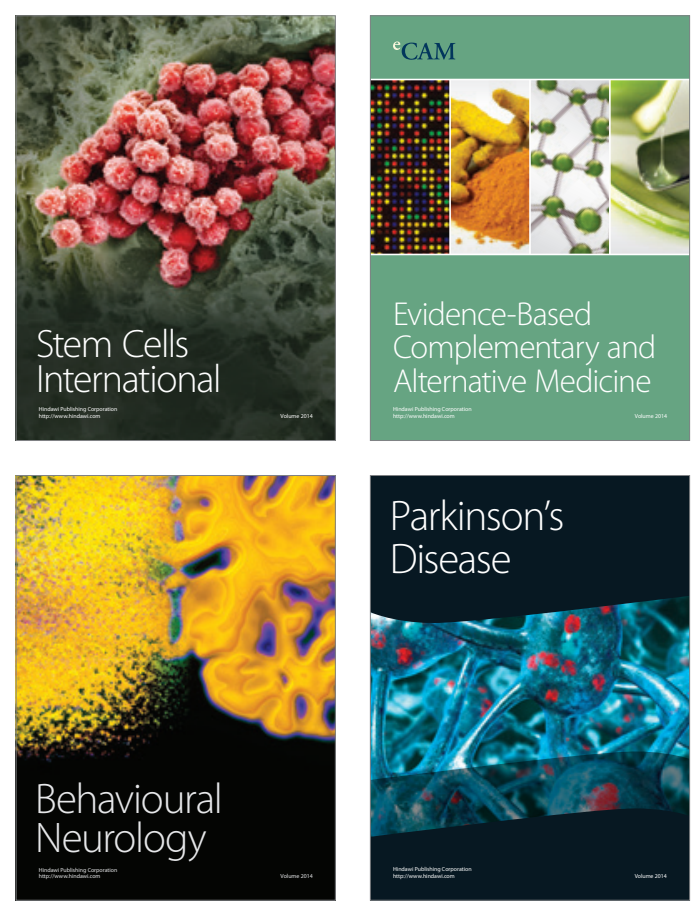

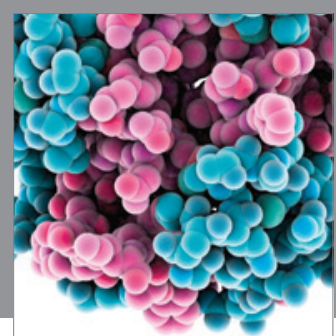

Journal of
Diabetes Research

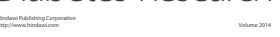

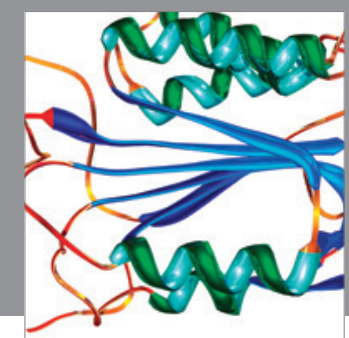

Disease Markers
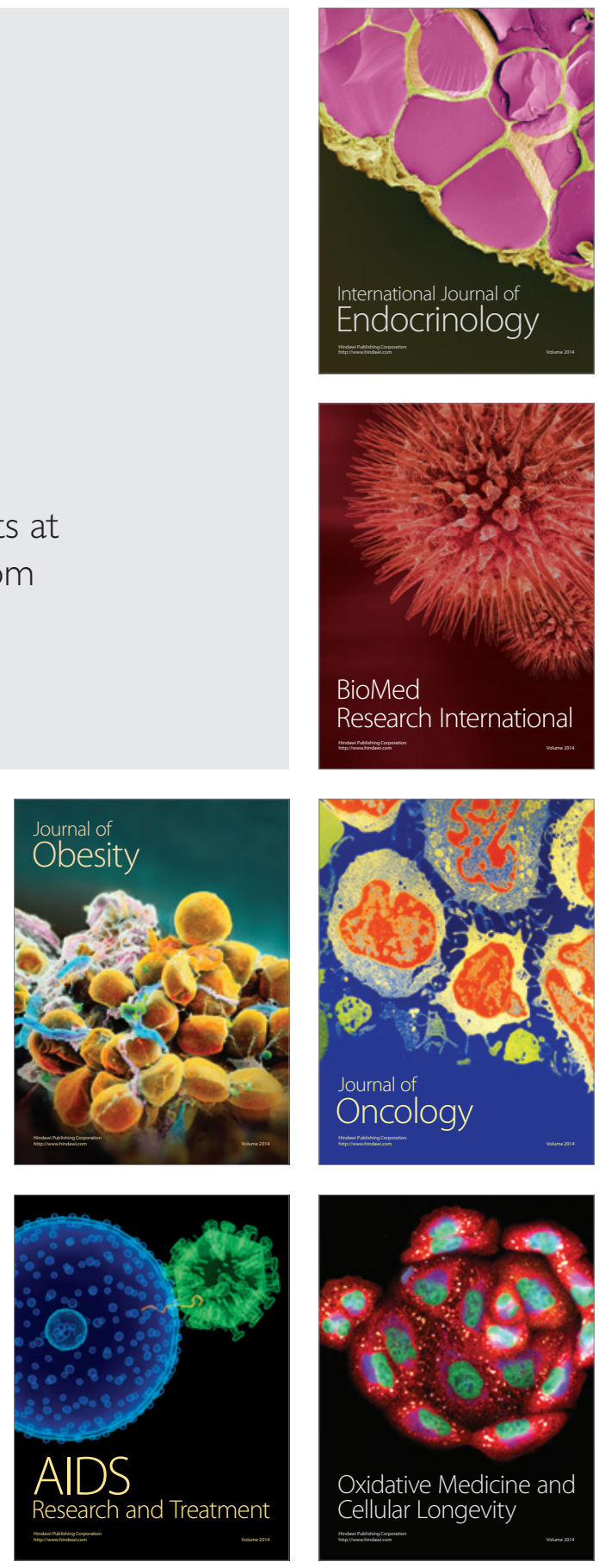